\title{
Hearing impairment risk high with diabetes
}

Published online: 16 November 2012

(C) Springer Healthcare 2012

medwireNews: People with diabetes are at high risk for developing a hearing impairment, report researchers.

Moreover, the association is likely to be independent of aging or a noisy environment, report Hirohito Sone (Niigata University Faculty of Medicine, Japan) and colleagues.

"It is believed that, over time, high blood glucose levels can damage the vessels in the stria vascularis and nerves, diminishing the ability to hear," explains the team in the Journal of Clinical Endocrinology and Metabolism.

The meta-analysis included 13 cross-sectional studies including 20,194 participants and 7377 patients with diabetes who had a hearing impairment, as assessed by pure-tone audiometry that included at least $2 \mathrm{kHz}$ of frequency range.

The team reports that the overall pooled odds ratio for hearing impairment among people with diabetes versus individuals without diabetes was 2.15, and the level of heterogeneity among studies was low enough to support a highly significant association

The association between hearing impairment and diabetes was strongest among younger individuals, aged 60 years or under, who had a 2.6-fold greater odds of hearing impairment than their nondiabetic counterparts, but the effect still remained significant in the older individuals.

The association was not significantly influenced by gender or chronic exposure to noisy environments either.

It has previously been reported that patients treated with insulin or hyperglycemic agents had thickened and atrophied vessel walls in the stria vascularis compared with those in healthy individuals, say Sone et al.

"Additional studies are needed to clarify the relationship between diabetes severity and prevalence of hearing impairment and the effect of glycemic control on hearing loss," they add.

By Sally Robertson, medwireNews Reporter

\section{Reference}

J Clin Endocrin Metab 2012; Advance online publication 